\title{
Entrevista motivacional: una herramienta en el manejo de la obesidad infantil
}

\author{
A. Martínez Rubio ${ }^{a}$ B. Gil Barcenillab

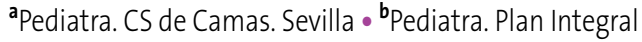 \\ de Obesidad Infantil de Andalucía. España.
}

La entrevista motivacional es un modelo de intervención en la consulta que ha mostrado su utilidad ante numerosas situaciones clínicas, especialmente en aquellos en que es necesario un cambio de conducta y es una alternativa más eficiente al consejo unidireccional.

Existen datos favorables de su aplicación en terrenos tan variados como: adicciones, diabetes, prevención del virus de la inmunodeficiencia humana, trastornos psiquiátricos, situaciones conflictivas, así como cuando es necesario proporcionar consejo dietético o preventivo.

El objetivo principal es que el paciente o la familia sean los protagonistas del cambio. Para ello se fundamenta en cuatro pilares básicos: mostrar empatía, detectar y aceptar la ambivalencia, manejar las resistencias y reforzar la autoeficiencia.

Los profesionales que deseen aplicar este modelo deben adquirir competencias específicas de comunicación, especialmente aquellas que han demostrado su utilidad para fortalecer la relación terapéutica y para resolver la ambivalencia.

Aplicado a la obesidad infantil, un momento especialmente crucial es el de la comunicación del diagnóstico, ya que el etiquetado suele generar resistencia, por lo que se aconseja emplear expresiones que muestren sensibilidad hacia las emociones del paciente y su familia.

\section{INTRODUCCIÓN}

La obesidad infantil, en un momento en que el $45,2 \%$ de los menores españoles de seis a diez años tienen sobrepeso $u$ obesidad ${ }^{1}$, es motivo de preocupación creciente entre el conjunto de profesionales sanitarios que atienden a la infancia. Hacer frente a este problema desde el espacio clínico implica reflexionar sobre las diferentes estrategias que pueden ser más efectivas de cara a su control.

El modelo médico tradicional aplica por lo general una fórmula básica bastante sencilla que la mayoría de los profesionales conoce y maneja de forma casi automática: tras obtener una serie de datos mediante la anamnesis y la exploración, precisándose o no algunas pruebas complementarias, se llega a un diagnóstico que se sigue de unas recomendaciones, que habitualmente se emiten en forma de prescripción. Lo más habitual es que se trate de algún fármaco, acompañado o no de algunas recomendaciones sobre la dieta y el régimen de vida.

Sin embargo, en el manejo de las enfermedades actuales relacionadas con los estilos de vida (hábitos alimentarios, sedentarismo, obesidad, consumo de tabaco o alcohol y otras adicciones) este modelo de abordaje es poco efectivo y produce, por 
ello, frustración tanto entre los pacientes como en los profesionales.

Como la obesidad en la infancia es un problema complejo desde su origen ya que interaccionan diversos factores genéticos $\mathrm{y}$, sobre todo, ambientales que condicionan los estilos de vida, también es complejo en su abordaje clínico y comunitario.

La realidad es que, a pesar de la evidencia disponible sobre la eficacia de las intervenciones para el control de la obesidad, no es raro encontrar, por un lado, bajos resultados en familias y pacientes, y por otro, desencanto e impotencia en los profesionales. Es como si hubiera un conjunto de "prejuicios" difusos en la sociedad, en las familias, y también entre los profesionales que dificultan trabajar de forma eficaz este problema de salud.

Estos "prejuicios" que se difunden por los medios de comunicación, están muy extendidos entre las familias e incluso entre los sanitarios. Louise Baur, una pediatra australiana, lo ha denominado Síndrome de resistencia a la obesidad ${ }^{2}$ al encontrarse con compañeros que muestran desinterés, antipatía, e incluso completa resistencia a trabajar con pacientes con obesidad.

Habría que preguntarse ¿Por qué la obesidad despierta emociones negativas tan fuertes entre algunos clínicos? ¿Se trata quizás de una aceptación poco meditada de algunos prejuicios existentes en la población sobre la obesidad? De hecho, algunos profesionales ven la obesidad como el resultado de un fallo moral (una mezcla de glotonería, pereza y falta de responsabilidad). Otros tienen una especie de nihilismo terapéutico: el tratamiento no funciona, entonces, ¿por qué preocuparse? Finalmente, otros están ansiosos si tienen que atender a chicos con obesidad pues saben que tienen poca o ninguna formación en esta área ${ }^{3}$.

La formación en el abordaje de la obesidad, al cambiar y mejorar la percepción sobre la efectividad de nuestras intervenciones ${ }^{4}$ y alejarse de los prejuicios, es uno de los caminos a recorrer para tratar de forma eficaz a pacientes y familias. Capacitarse para utilizar las intervenciones más eficaces y adoptar un estilo de trabajo más acorde con la naturaleza del problema -centrado en la comunicación- puede ser una buena forma de enfrentarse a la obesidad infantil.

\section{EL ESTILO DE COMUNICACIÓN EN LA CONSULTA PEDIÁTRICA EN RELACIÓN CON LA OBESIDAD}

La obesidad es una enfermedad crónica estrechamente relacionada con los estilos de vida y, como tal, precisa un abordaje similar al de otras enfermedades crónicas: diagnóstico, tratamiento orientado al cambio de hábitos alimentarios y sobre actividad física y seguimiento para evitar las recaídas. Probablemente, los pacientes pasarán por periodos de avances y retrocesos y por etapas de mejor control. Los profesionales necesitan ajustar su práctica clínica a los ritmos de pacientes y familias evitando la confrontación para no generar resistencias.

Siempre hay que tener en cuenta la importancia del soporte familiar, educativo y social de los pacientes sin perder de vista la perspectiva de género en este problema. A pesar de que los problemas de peso son más frecuentes entre los chicos que entre las chicas, la realidad es que hay más presión social para que las chicas adelgacen y, por otro lado, muchos chicos -y sus familias - no son suficientemente conscientes del exceso de peso y no se están tratando adecuadamente.

En esta enfermedad es importante manejar adecuadamente algunos aspectos clave que tienen que ver esencialmente con el estilo de comunicación que se establece entre profesionales, pacientes y familias para ayudarles de forma eficaz a incorporar estilos de vida saludables en alimentación y en actividad física sin generar resistencias. Es un formato no directivo centrado en el trabajo sobre la motivación y que utiliza como herramienta la entrevista motivacional (EM). Está demostrado que adoptando un enfoque clínico que se centra más en la escucha reflexiva y en el apoyo como la EM se pueden mejorar la implicación de los pa- 
cientes y la respuesta al tratamiento, incluso en entrevistas breves.

Los modelos de tratamiento basado en la EM nacieron de la necesidad de encontrar herramientas adecuadas para el manejo de pacientes afectos de adicciones, a quienes era necesario fortalecer para que pudieran llegar a ser protagonistas de cambios sostenidos en sus estilos de vida y reforzar sus estrategias de afrontamiento. También se han aplicado para la prevención de conductas de riesgo, en el tratamiento de la diabetes y de otras enfermedades crónicas y, cada vez más para el manejo de la obesidad ${ }^{5}$.

Para lograr una relación terapéutica más eficaz es aconsejable que esté centrada en el paciente, construyendo una atmósfera segura y de apoyo que le permita encontrar sus propias soluciones. Las claves están en la empatía, la calidez y la autenticidad ${ }^{6}$.

Los estadios del cambio explican por qué en ciertos momentos es más probable que una persona abandone un hábito y cuáles son las señales de que se encuentra preparada. La clave está en la motivación, y por ello, la tarea principal del terapeuta es ayudar al paciente a encontrar sus propios motivos y razones, a seguirlos, a superar la ambigüedad, identificando y sorteando los obstáculos $^{7}$.

\section{COMUNICAR EL DIAGNÓSTICO CON SENSIBILIDAD}

Los profesionales sanitarios nos hemos especializado en realizar diagnósticos. Es un terreno en que nos sentimos a gusto, ya que fue y es la base de la formación médica tradicional. Sin embargo, a veces no somos conscientes del impacto emocional que las etiquetas tienen en los pacientes y sus familias.

Los menores y sus familias suelen vivir el problema del exceso de peso de forma problemática y el tema de cómo comunicar el diagnóstico de obesidad es un asunto complejo. Dependiendo de qué palabras se usen, los pacientes y sus familias van a estar más o menos proclives a abordar el problema y a avanzar en sus soluciones.

Dutton $^{8}$ estudió este asunto, analizando las preferencias de los pacientes y los términos más utilizados por los profesionales. Los pacientes señalaron que preferían que se utilizara la palabra "peso", mientras que "obesidad" era el término que menos les gustaba. En ese estudio los médicos declararon que procuraban adaptarse a las preferencias de los pacientes.

\section{FUNDAMENTOS DE LA ENTREVISTA MOTIVACIONAL}

Los pediatras que deseen incorporar la EM en la práctica deben desarrollar estrategias de comunicación que han demostrado su utilidad para fortalecer la relación terapéutica y para resolver la ambivalencia.

La EM se basa en expresar empatía, aceptar la ambivalencia, manejar las resistencias y reforzar la autoeficiencia.

La relación terapéutica debe basarse en la aceptación incondicional de la persona y desarrollarse en un clima de empatía y escucha activa como ingredientes esenciales para favorecer que el paciente descubra sus propias motivaciones, y se oiga a sí mismo decirlas y de ese modo se convierta en protagonista y autor de sus decisiones.

El comportamiento es el resultado de ideas y sentimientos. Por eso, para cambiar las conductas es necesario abordar tanto las ideas como las emociones. Se trata de identificar lo que mantiene las conductas, incluyendo la ambivalencia ("quiero pero no quiero") y esto se logra mejor aceptando los puntos de vista del paciente y evitando la confrontación.

La EM es un enfoque centrado en el paciente y la familia, que pretende la toma de decisiones de forma colaborativa, proporciona feed-back sin enjuiciar, acepta la resistencia al cambio por parte del paciente y les anima a desarrollar sus propios motivos para el cambio de conducta de salud $d^{9,10}$ 


\section{HERRAMIENTAS ÚTILES EN LA ENTREVISTA MOTIVACIONAL}

\section{Pedir permiso}

En nuestra habitual postura de "autoridad médica" y tras unos cuantos años de trabajar a contrarreloj en las consultas, parece que a los médicos nos está permitido olvidar ciertas normas elementales de cortesía. Tenemos prisa y los interrogatorios se hacen de forma intrusiva. Por eso y por falta de formación, tampoco solemos indagar acerca de las expectativas y creencias acerca de la salud o las enfermedades.

Un punto clave de la entrevista motivacional es el respeto a la autonomía del paciente. Por eso, es recomendable solicitar permiso ${ }^{11,12}$ para abordar el tema del "peso" del menor, así como otros temas en que vayamos a utilizar este modelo de entrevista.

¿Les parece buen momento ahora para hablar acerca del peso de Juan?

¿Cuándo les parece que nos reunamos para tratar del tema de Adela y su alimentación?

Cuando Vds. lo consideren conveniente nos vemos de nuevo para comentar las posibilidades que ven para motivar a Jaime.

A menudo sentimos la urgencia de hacer algo en seguida, sin tener en cuenta si la familia está o no preparada. Ese furor curandi suele estar abocado al fracaso llevando al profesional a la frustración.

Además, esta forma de comenzar significa entregar el timón a la familia. Son ellos, solo ellos quienes tienen que hacer los ajustes necesarios. Pedirles el permiso es concederles el poder de cambiar desde el primer momento.

\section{Escucha reflexiva}

Esto no quiere decir escuchar en silencio reflexionando o haciendo hipótesis para uno mismo, sino que tras cada respuesta del paciente se le devuelve una reflexión o un resumen, para comprobar si es eso lo que quiere decir ${ }^{9,11,12}$. Buscando el significado detrás de las palabras del paciente. Construyen- do hipótesis en lugar de asumir de forma pasiva. Esa devolución, en forma de pregunta evita resistencias y construye un entorno de aceptación, sin enjuiciamiento y además abre perspectivas. La reflexión se usa para reforzar algunos aspectos de lo que la persona ha dicho o para alterar su significado levemente. Puede hacerse de varias formas:

- Reflexión simple: repetir lo que el paciente acaba de decir con palabras similares.

- Reflexión sobre el significado: recoger el contenido cognitivo de lo que acaba de expresar.

- Reflexión sobre el sentimiento: reflejar el contenido emocional de lo que dice el paciente.

- Reflexión doble: se usa para poner de manifiesto los dos lados de la ambivalencia empezando por el lado que favorece mantener la conducta de partida y terminando por el lado inclinado hacia el cambio.

- Reflexión amplificada: se usa cuando solo se ha manifestado el lado negativo de la ambivalencia exagerándolo con cuidado de no caer en el sarcasmo. Esto conduce a que el paciente desee corregir la distorsión.

Se pueden ver algunos ejemplos en la Tabla 1.

\section{Hacer preguntas abiertas}

Cuando disponemos de pocos minutos para una consulta, nos hemos habituado a emitir ráfagas de preguntas. Sin embargo, el si o el no representan posturas demasiado antagónicas que impiden la reflexión y el avance. Además, las preguntas que pueden responderse con pocas palabras mantienen el modelo de "experto activo-paciente pasivo".

Aprender a hacer preguntas abiertas no es difícil aunque requiere un poco de entrenamiento. En el enfoque de la EM este debe ser el formato habitual. A veces ni siquiera tienen el formato de pregunta.

Entre toda la familia ¿quién es la persona que más se preocupa por la salud de Juan?

Me gustaría saber algo más acerca de las costumbres de su familia los fines de semana. 


\begin{tabular}{|c|c|c|c|}
\hline $\begin{array}{l}\text { El paciente/la familia } \\
\text { dice }\end{array}$ & El terapeuta dice & Técnica que ha usado & Que consiste en \\
\hline $\begin{array}{l}\text { Es que no me gustan } \\
\text { las verduras (Andrés, } 13 \\
\text { años) }\end{array}$ & $\begin{array}{l}\text { O sea, que no te } \\
\text { imaginas a ti mismo } \\
\text { probando una ensalada }\end{array}$ & Reflexión simple & $\begin{array}{l}\text { Repetir lo que el paciente acaba } \\
\text { de decir, con palabras parecidas }\end{array}$ \\
\hline Yo no estoy TAN gordo & $\begin{array}{l}\text { O sea que la etiqueta de } \\
\text { "gordo" no te gusta }\end{array}$ & $\begin{array}{l}\text { Reflexión sobre el } \\
\text { significado }\end{array}$ & $\begin{array}{l}\text { Añade el contenido cognitivo que } \\
\text { subyace en la frase que ha dicho }\end{array}$ \\
\hline $\begin{array}{l}\text { Yo no estoy TAN gordo } \\
\text { para que me llamen "el } \\
\text { albóndiga" en el cole }\end{array}$ & $\begin{array}{l}\text { Parece que te sientes mal } \\
\text { cuando se meten contigo } \\
\text { por tu aspecto }\end{array}$ & $\begin{array}{l}\text { Reflexión sobre las } \\
\text { emociones }\end{array}$ & $\begin{array}{l}\text { Reflexión sobre el contenido } \\
\text { afectivo o las emociones } \\
\text { implicadas en la frase dicha }\end{array}$ \\
\hline $\begin{array}{l}\text { No necesito comer } \\
\text { ensalada para estar más } \\
\text { delgado. Estoy bien así }\end{array}$ & $\begin{array}{l}\text { Te gustas tal y como eres } \\
\text { ahora. Te sientes bien con } \\
\text { tu cuerpo }\end{array}$ & Reflexión amplificada & $\begin{array}{l}\text { Se usa cuando solo se ha } \\
\text { expresado el lado negativo de } \\
\text { la ambivalencia. Se exagera o } \\
\text { intensifica de forma intencionada } \\
\text { para poner en evidencia la } \\
\text { distorsión. Usar con cuidado para } \\
\text { no caer en el sarcasmo }\end{array}$ \\
\hline $\begin{array}{l}\text { Es que estoy harto de } \\
\text { que mi madre insista } \\
\text { tanto en que coma toda } \\
\text { clase de verduras. Ya le he } \\
\text { dicho que me gustan los } \\
\text { guisantes, las lentejas y } \\
\text { el gazpacho pero que no } \\
\text { me gustan las ensaladas } \\
\text { crudas ni la coliflor }\end{array}$ & $\begin{array}{l}\text { Me parece que quieres } \\
\text { decirnos que te sientes } \\
\text { mal cuando tu madre te } \\
\text { sermonea sobre lo que } \\
\text { debes comer porque ya } \\
\text { has hecho esfuerzos para } \\
\text { tomar más verduras }\end{array}$ & Reflexión doble & $\begin{array}{l}\text { Refleja las dos perspectivas } \\
\text { de la ambivalencia expresada. } \\
\text { Es preferible comenzar con el } \\
\text { lado que favorece seguir igual } \\
\text { y terminar con el favorable al } \\
\text { cambio }\end{array}$ \\
\hline
\end{tabular}

Quizá me puedan explicar detalladamente qué ocurre cuando Adela empieza a hacer excursiones a la nevera.

Me gustaría que me contaran como organizan la tarde para Jordi cuando Vds. tienen que trabajar. ¿Qué es lo que más le preocupa en el actual esquema de alimentación de Vanesa?

\section{Detectar la predisposición al cambio}

Iniciar los esfuerzos terapéuticos o lanzarse a hacer recomendaciones sin saber si la familia está dispuesta para iniciar cambios en su vida suele conducir a la frustración y al fracaso.

La EM se sustenta en el modelo transteorético de predisposición al cambio de Prochaska y Di Clemente ${ }^{13}$.

Los estadios del cambio explican por qué en ciertos momentos es más probable que una persona abandone un hábito y cuáles son las señales de que se encuentra preparada para cambiar. La clave está en la motivación, y por ello, la tarea principal del terapeuta es ayudar al paciente a encontrar sus propios motivos y razones, a seguirlos, a superar la ambigüedad, identificando y sorteando los obstáculos ${ }^{13}$.

En la Tabla 2 se ejemplifican conversaciones que permiten deducir en qué estadio se encuentran. En cada etapa el clínico tiene distintos objetivos y por eso adoptará estrategias diferentes, desde posponer la conversación a otro momento a continuar explorando, proponer tareas, etc.

\section{Trabajar con la ambivalencia}

En muchas conductas humanas está presente la ambivalencia (quiero y a la vez no quiero hacerlo). Hacer excesivo hincapié en uno de los lados de la balanza suele conducir a que el paciente se posicione en el lado contrario. Sin embargo, destacar las partes del discurso del paciente que reflejan su doble posición, sin juzgarle, le permitirá tomar conciencia de los motivos que subyacen a la conducta. 
Tabla 2. Objetivos y estrategias en cada una de las etapas del cambio. Algunas frases que sugieren el estadio

\begin{tabular}{|c|c|c|c|}
\hline Conversación & Estadio & Objetivos & Actitud del profesional \\
\hline $\begin{array}{l}\text { "Sí, Sara siempre ha sido grandecita, } \\
\text { como toda mi familia" } \\
\text { "Creo que Carlos es aun muy } \\
\text { pequeño para hacer una dieta. } \\
\text { Además, seguro que cuando de el } \\
\text { estirón de la pubertad adelgazará" }\end{array}$ & Precontem-plación & $\begin{array}{l}\text { Ayudar a que tomen la } \\
\text { decisión por sí mismos/as } \\
\text { (inclinar la balanza) }\end{array}$ & $\begin{array}{l}\text { Evocar las razones a favory } \\
\text { en contra } \\
\text { Aumentar la autoeficacia } \\
\text { para el cambio }\end{array}$ \\
\hline $\begin{array}{l}\text { "Bueno, estamos preocupados } \\
\text { porque Celia esta un poco gordita. } \\
\text { Pero no sabemos bien si es } \\
\text { adecuado hacer algo ahora" }\end{array}$ & Contemplación & $\begin{array}{l}\text { Reforzar su decisión de } \\
\text { cambio }\end{array}$ & $\begin{array}{l}\text { Identificar obstáculos y } \\
\text { estrategias para afrontarlos } \\
\text { Ofrecer recursos y apoyo }\end{array}$ \\
\hline $\begin{array}{l}\text { "Hemos hablado con Manuel. El } \\
\text { también cree que debe ponerse } \\
\text { más en forma para sentirse mejor } \\
\text { cuando va a la piscina con su } \\
\text { pandilla" }\end{array}$ & Preparación & $\begin{array}{l}\text { Ayudar a dar los pasos } \\
\text { hacia el cambio }\end{array}$ & $\begin{array}{l}\text { Identificar obstáculos y } \\
\text { estrategias para afrontarlos } \\
\text { Ofrecer recursos y apoyo }\end{array}$ \\
\hline $\begin{array}{l}\text { "Estamos contentos con los } \\
\text { progresos de Isabel estos últimos } \\
\text { dos meses. Nos preocupa un poco } \\
\text { qué pasará en las vacaciones de } \\
\text { verano" }\end{array}$ & Acción & $\begin{array}{l}\text { Mantener el cambio y/o } \\
\text { prevenir la recaída }\end{array}$ & $\begin{array}{l}\text { Reforzar los logros } \\
\text { obtenidos: felicitar } \\
\text { Distinguir recaída y desliz } \\
\text { (temporal y reversible) } \\
\text { Identificar disparadores de } \\
\text { crisis y cómo afrontarlos } \\
\end{array}$ \\
\hline $\begin{array}{l}\text { "El verano ha sido un desastre. } \\
\text { Todos hemos bajado la guardia" }\end{array}$ & Recaída & $\begin{array}{l}\text { Destacar el esfuerzo } \\
\text { realizado y valorar la } \\
\text { motivación para intentarlo } \\
\text { de nuevo }\end{array}$ & $\begin{array}{l}\text { Identificar los motivos de la } \\
\text { recaída y cómo superarlos } \\
\text { Sugerir alternativas de } \\
\text { apoyo y afrontamiento }\end{array}$ \\
\hline
\end{tabular}

La ambivalencia puede ser muy confusa, frustrante y difícil de comprender tanto por el paciente como por el terapeuta, entre otras cosas porque puede ser cambiante y muchos de sus aspectos son inconscientes e irracionales. También influyen los valores y expectativas de la persona y se ve matizada por la autoestima y el contexto social.

Un recurso muy utilizado en la EM es la "regla", una especie de escala para que el paciente indique cuán cercano está a una de las posiciones (a favor o en contra del cambio). Una vez que se posiciona, el terapeuta debe cuestionar, "tirando hacia abajo" de la fortaleza de su decisión para estimular que continúe aportando argumentos a favor del cambio.

Por el contrario, no conviene caer en la trampa de asumir la parte "a favor del cambio" pues el paciente entonces se posicionará en el lado opuesto. El clínico debe mantenerse cerca de los sentimientos, valores y creencias del paciente mediante sus ha- bilidades empáticas y técnicas específicas que detallaremos más adelante.

"Ponga puntuación del 1 al 10 de cuánto cree Vd. que está dispuesto a cambiar su forma de hacer la compra..." Luego se puede ayudar al paciente a tomar más conciencia con la técnica del "regateo" o de las diferencias. "Vd. dijo que 5. ¿Por qué no 4? O bien ¿Cuál es la diferencia con el 6? ¿Como es que lo ve Vd. tan claro que es un 5 ?" Esto va a permitir descubrir nuevos obstáculos o barreras.

Puede ser interesante que la familia o el paciente hagan su propia lista de argumentos a favor o en contra del cambio referido a conductas concretas.

Por su parte, el profesional puede intervenir señalando las discrepancias y contradicciones entre los deseos y valores y la conducta, entre lo que hace y lo que le gustaría hacer. No se trata de sermonear o razonar o convencer, sino de favorecer que el paciente se escuche a sí mismo desarrollando sus propios argumentos para cambiar de estilo de vida. 


\section{Saber manejar las resistencias}

Cuanto más se resiste el paciente, menos probabilidades hay de que cambie. En el enfoque de EM se considera que la resistencia del paciente es un problema del terapeuta. Por eso es necesario aprender a discernir las expresiones que indican resistencia (Tabla 3) y si se detectan, cambiar la estrategia. Es conveniente evitar frases y actitudes de tipo autoritario, paternalista, conductivo o confrontativo ya que estos enfoques tienden a despertar resistencias en los pacientes (Tabla 4).

\section{Ofrecer alternativas}

Las reflexiones del terapeuta ante la ambivalencia deben servir para mostrar al paciente o a la familia los diversos aspectos de las conductas, las caras ocultas de sus resistencias y también mostrar posibles soluciones. Sin embargo la decisión final la tienen ellos. Debe quedar claro que el paciente es libre para elegir, que si escoge no cambiar está en su derecho. El terapeuta tiene que animar al cambio pero no insistir en el cambio.

\section{Favorecer la autoeficacia y conseguir que expresen afirmaciones automotivadoras}

La motivación puede ser definida como la probabilidad de que una persona inicie, continúe y se com- prometa con una estrategia específica para cambiar $^{9,10}$

La motivación depende del contexto. Una persona puede "estar motivada" o no, pero también puede "ser motivada". Lograrlo es una parte importante de la tarea del terapeuta. Cuando la persona se oye decir a sí misma que es capaz, que quiere cambiar de hábitos se encuentra mucho más cerca del cambio real. Para lograrlo se recurre a algunas de las estrategias comunicacionales ya comentadas.

\section{Proporcionar feed-back}

La motivación se produce cuando la persona percibe una discrepancia entre el momento en que se encuentra y el lugar a donde quiere llegar. Puede ser facilitado al compartir el resultado de pruebas, pero permitiendo que el paciente pueda formarse sus propias conclusiones y dejando clara la libertad para elegir. Hay varias herramientas para ello. Una de las más utilizadas el la "hoja de balance" en que el paciente anota las ventajas e inconvenientes tanto de la conducta a cambiar como de su alternativa. El terapeuta debe estar atento a la comunicación no verbal y al contenido emocional del discurso del paciente. Devolver al paciente sus propias afirmaciones automotivadoras es también una poderosa herramienta para el feed-back.

\begin{tabular}{|c|c|}
\hline $\begin{array}{l}\text { Argumentar. El paciente cuestiona la habilidad, el nivel de } \\
\text { conocimientos o la integridad del terapeuta }\end{array}$ & $\begin{array}{l}\text { - Desvalorizar } \\
\text { - Retar } \\
\text { - Hostilidad }\end{array}$ \\
\hline $\begin{array}{l}\text { Interrumpir. El paciente corta o interrumpe al terapeuta de manera } \\
\text { defensiva }\end{array}$ & $\begin{array}{l}\text { - Discutir } \\
\text { - Cortar }\end{array}$ \\
\hline $\begin{array}{l}\text { Negar. El paciente expresa no querer reconocer los problemas o } \\
\text { cooperar o aceptar la responsabilidad o aceptar un consejo }\end{array}$ & $\begin{array}{l}\text { - Culpabilizar } \\
\text { - Estar en desacuerdo } \\
\text { - Reclamar impunidad } \\
\text { - Minimizar } \\
\text { - Pesimismo } \\
\text { - Reticencia } \\
\text { - No querer cambiar }\end{array}$ \\
\hline Ignorar. El paciente da señales de no seguir o ignorar al terapeuta & $\begin{array}{l}\text { - Falta de atención } \\
\text { - Falta de respuestas } \\
\text { - No respuesta } \\
\text { - Desviarse }\end{array}$ \\
\hline
\end{tabular}


Tabla 4. Tipos de respuestas que deben evitarse ya que bloquean la comunicación y dificultan el cambio. Todas implican una relación desigual

- Ordenar, dirigir o encargar

- Preocupar, amenazar

- Aconsejar, sugerir soluciones, realizar sugerencias

- Persuadir, discutir, argumentar, convencer

- Moralizar, predicar, sermonear, decir al paciente lo que debería hacer

- Estar en desacuerdo, juzgar, acusar, culpabilizar

- Estar de acuerdo, aprobar, rogar

- Avergonzar, ridiculizar, etiquetar

- Interpretar, analizar

- Reafirmar, consolar, simpatizar

- Cuestionar o poner a prueba

- Retirarse, distraerse, hacer una broma o cambiar de tema

\section{CONCLUSIONES}

Las técnicas de consejo habitualmente se limitan a hacer recomendaciones y observaciones concretas sobre conductas concretas, sin embargo es fácil que choquen con factores emocionales, creencias o las expectativas de los pacientes. La principal barrera emocional es la ambivalencia que se siente ante una conducta hacia la que se ha creado cierto grado de adherencia o adicción pero que a la vez se desea abandonar por sus consecuencias. Dentro de esos mismos polos también suele haber ambivalencia.

La EM es una forma de terapia que se basa en la conversación y el lenguaje. Puede ser aprendida por quien desee ayudar a otras personas a encontrar nuevas maneras de cambiar aspectos de su comportamiento ${ }^{14}$. No es rápido ni fácil de apren- der porque supone un cambio radical en la práctica en las consultas médicas habituales. Sin embargo, una vez aprendido, se muestra de gran utilidad en el manejo de las enfermedades crónicas, las adicciones, los cambios de hábitos, etc. Además, el estilo comunicacional y las habilidades específicas de la EM son de utilidad en muchas situaciones cotidianas de la clínica (manejo de resistencias en general, de situaciones conflictivas, adherencia al tratamiento...). Vale la pena entrenarse y aprovechar esta interesante herramienta de trabajo.

\section{CONFLICTO DE INTERESES}

Las autoras declaran no presentar conflictos de intereses en relación con la preparación y publicación de este artículo.

\section{ABREVIATURAS}

EM: entrevista motivacional. 


\section{BIBLIOGRAFÍA}

1. Agencia Española de Seguridad Alimentaria y Nutrición. Ministerio de Sanidad, Servicios Sociales e Igualdad. Estudio de prevalencia de obesidad infantil "ALADINO" (ALimentación, Actividad física, Desarrollo INfantil y Obesidad). 2011 [en línea]. Disponible en www.naos.aesan.msps.es/naos/investigacion/ aladino/

2. Baur LA. Changing perceptions of obesity-recollections of a paediatrician. The Lancet. 2011;378(9793): 762-3.

3. Tanas R. Curso de formación de pediatras, 2012. Comunicación personal de la autora.

4. Barroso Espadero D. Componentes de las intervenciones para tratar la obesidad y el sobrepeso infantil desde la Atención Primaria: revisión sistemática de efectos. Evid Pediatr. 2012;8:26.

5. Centre of Reviews and Dissemination (CRD). Components of primary care interventions to treat childhood overweight and obesity: a systematic review of effect. University of York. Database of Abstracts of Review of Effects web site (DARE). Documento número: 12010003052 [en línea] [actualizado en 2011; consultado el 12/12/2011]. Disponible en www.crd.york.ac.uk/crdweb/ShowRecord. asp? AccessionNumber $=12011002915 \&$ User ID $=144$

6. Hassink S. Obesidad infantil. Prevención, intervenciones y tratamiento en atención primaria. Madrid: 2007.
7. Rogers CR. The necessary and sufficient conditions of therapeutic personality change. J Consult Psychol. 1957;21(2):95-103.

8. Prochaska JO, DiClemente CC. Stages and processes of self-change of smoking: toward an integrative model of change. J Consult Clin Psychol. 1983;51(3):390-5.

9. Dutton GR, Tan F, Perri MG, Stine CC, Dancer-Brown $M$, Goble $M$, et al. What words should we use when discussing excess weight? J Am Board Family Med. 2010;23(5):606-13.

10. Rollnick S, Miller WR, Butler CC. Motivational intervieweing in health care. Helping patients to change behavior. New York-London: The Gilford Press; 2008.

11. Miller WR, Rollnick S. La entrevista motivacional. Preparar para el cambio de conductas adictivas. Barcelona-Buenos Aires: Ediciones Paidós Ibérica S.A.; 1999.

12. Gold MA, Kokotailo PK. Motivational interviewing strategies to facilitate adolescent behavior change. Pediatrics. 2007;20(1):1-10.

13. Barnes AJ, Gold MA. Promoting healthy behaviors in pediatrics: Motivational interviewing. Pediatrics Rev. 2012;33(9):e57-e68.

14. Prochaska JO, DiClemente CC. Stages and processes of self-change of smoking: Toward an integrative model of change. J Consulting Clin Psychology. 1983;51(3): 390-5.

15. Arroba Basanta ML, Dago Elorza R. Metodología del consejo. Estrategias que favorecen la adquisición o el cambio de hábitos de los pacientes y sus padres. Rev Pediatr Aten Primaria. 2008;10(Sup 2):e45-55. 